\title{
La construcción de un gatekeeper
}

\section{El caso de Roger Caillois (1939-1951)}

El objetivo de mi intervención es volver sobre el concepto de gatekeeper que usamos para pensar la circulación internacional de literatura, a partir de una reflexión sobre el caso de Roger Caillois, quien, como es sabido, jugó un papel determinante en la traducción y difusión de la literatura hispanoamericana en Francia. De modo más ambicioso, quisiera intentar volver sobre el uso que los especialistas de literatura hacemos de conceptos que provienen del campo de la sociología y en especial de la sociología de la literatura; lo que se podría considerar el "telón de fondo" de este trabajo en curso es un cuestionamiento de la extensión de la teoría de Pierre Bourdieu sobre el funcionamiento del campo intelectual del nivel nacional al nivel internacional, generalmente aceptada (1966, 1991, Sapiro 2007).

Desde el punto de vista metodológico, mi aproximación, aunque toma prestados conceptos de la sociología, se diferencia de esta por el papel y la función que otorga a las producciones textuales. Como es sabido, Bourdieu define tres mediaciones que organizan el campo literario: las condiciones materiales de producción y de circulación de las obras; los modos de su producción por sus autores individuales y sociales; las condiciones de su recepción. A estas mediaciones, propongo agregar los posicionamientos, modelos y representaciones que proponen los textos mismos, que se ubican en el centro del análisis, lo cual reorganiza las relaciones entre los tres tipos de mediaciones propuestos por Bourdieu, y permite pensar lo escrito como una fuerza activa, que puede imponerse sobre las otras condiciones. Conviene, además, recordar que la teoría del campo de Bourdieu tenía como objetivo sobrepasar el clivaje entre una aproximación interna y una externa de las condiciones culturales: el análisis interno se focaliza en el desciframiento, más que en el acto creador, y se interesa en la estructura de las obras; el análisis externo se interesa en la función social de las obras. A pesar de ello, notamos que, a menudo, la sociología de la literatura, cuando se trata en particular de "world editing" o simplemente de "editing”, otorga poca atención a las obras mismas y a su inscripción en tradiciones literarias.

A partir de estos principios, quisiera proponer un análisis de algunos agentes y factores que solemos reunir bajo el término de gatekeeper en el campo de

Annick Louis, Université de Reims/CRIMEL CRAL, EHESS-CNRS

Ә Open Access. ( 2021 Annick Louis, published by De Gruyter. (c) BY-NC-ND This work is licensed under a Creative Commons Attribution-NonCommercial-NoDerivatives 4.0 International License.

https://doi.org/10.1515/9783110713015-017 
los estudios de la traducción, que experimenta actualmente un desarrollo importante en el área latinoamericana. Como Marcelo Topuzian pienso que la noción de literatura mundial propone un marco conceptual que permite - o debería permitir - revisar "las segmentaciones institucionales todavía vigentes en los estudios literarios a partir de su ‘desespecificación' tanto espacial o geográfica como temporal o epocal pero también teórica y epistemológica” (2014: 1). Mi uso del condicional viene del hecho que en Francia esta posibilidad es, por el momento, enteramente virtual; aunque numerosos investigadores hemos comenzado a reorganizar nuestros objetos de investigación, en el nivel institucional seguimos observando una segmentación importante de las disciplinas y subdisciplinas - no necesariamente favorable a una adaptación de nuestras instituciones a los avances contemporáneos de la investigación. Pensar la circulación e inscripción de las traducciones de literatura extranjera en las historias literarias nacionales puede permitir no solamente resignificar el funcionamiento del medio, sino también proponer nuevos objetos e imaginar nuevas topografías académicas para los estudios literarios.

\section{Definición y conceptualización}

Conviene comenzar interrogándose acerca de la terminología. En español, solemos usar el término de "mediador" como equivalente de gatekeeper, pero sus significados difieren: la palabra inglesa acentúa el poder y la posición de quien ejerce esa función, mientras que el “mediador” puede aparecer como más neutro, como un simple intermediario casual. Estas diferencias de significado implican la necesidad de reflexionar acerca de la traducción del inglés, y muestran también que es necesario volver sobre la definición del término.

Fue Loren Glass quien conceptualizó el término gatekeeper en relación con los expertos literarios que regulan los vínculos entre autor y audiencia (2008); en inglés, el término remite a una persona que controla el acceso a algo, y, por tanto, puede tomar el significado de guardián, centinela, interrogador o juez; en el mundo de la edición, designa generalmente al consejero financiero y editorial, cuyos roles suelen ser proteger, seleccionar, controlar y editar. Es evidente que el término y su uso subrayan el hecho que cuando se identifica a una persona con la noción, es porque esta asume el rol de garante de una institución y legisla la admisión a esta, así como las condiciones en que se entra; pero también, como lo señala Judith Schlanger, controlando las admisiones tiene una influencia tal vez más importante que en tanto fuerza excluyente (1992). Doble función, por tanto, de protección y de selección, procesos en los que 
entran en juego diferentes factores, criterios personales, institucionales, financieros e ideológicos.

Recientemente, William Marling señaló con razón la ausencia de estudios sobre los motivos y procedimientos usados por los gatekeepers, es decir sobre los personajes concretos - editores, traductores, escritores-, y sus modos de funcionamiento; sin embargo, su trabajo confirma la importancia de estos agentes, término que designa a los diferentes actores envueltos en el pasaje de una zona cultural a otra y no solamente a los agentes literarios, aunque, como veremos, considero que actualmente estos juegan un papel que correspondería al concepto de gatekeeper (2016). Mi posición, sin embargo, coincide en parte con la crítica que hace John B. Thompson, en Merchants of Culture: The Publishing Business in the Twenty-First Century (2012), donde afirma que el concepto simplifica las formas complejas de la interacción y negociación entre autores, agentes y editores; pero Thompson ubica el capital en las "publishing firms" y no en los individuos. En este sentido su posición se asemeja a la de Ana Gallego Cuiñas, quien postula la necesidad de pensar el concepto en términos de dispositivo (2018). Ambos se basan en la conceptualización de Michel Foucault, que, a partir de los años 1970, piensa el dispositivo como un conjunto heterogéneo formado por discursos, instituciones, organizaciones arquitectónicas, reglas y leyes (1971, 1975). En otros términos, el dispositivo está representado por la red establecida entre estos diferentes elementos, y se inscribe siempre en una relación de poder; se constituye de lo dicho (discursos) y de lo no dicho (formas arquitecturales, por ejemplo). Por lo tanto, el dispositivo tiene una función esencialmente estratégica y se establece en general para responder a una urgencia; cambia permanentemente de posición, se adapta y se reajusta. El dispositivo es el lugar de inscripción de técnicas de un proyecto social, que actúa por coerción y apunta al control de los cuerpos y las mentes, lo que significa que influye en nuestros comportamientos. A esta conceptualización, quisiera agregar la observación de Michel de Certeau, quien subraya el hecho que Foucault permitió constituir los procedimientos y las técnicas en objetos, pero que se limitó a ciertos dispositivos técnicos; De Certeau señala que existe una vasta cantidad de prácticas técnicas mudas, consideradas "menores", que quedan por estudiar (1990: 79).

En la continuidad entonces de la conceptualización de Foucault de la noción de dispositivo y de su ajuste propuesto por De Certeau, y coincidiendo, como ya señalé, tanto con Thompson como con Gallego Cuiñas, trataré de considerar el papel, las funciones y las competencias de Roger Caillois a partir del concepto de dispositivo. Mi propuesta, sin embargo, consiste en abandonar el uso de un concepto único para designar el modo en que actúan ciertos agentes en el campo entre diferentes espacios culturales en el ámbito editorial; la razón 
principal es que asistimos actualmente a un uso indiscriminado del uso del concepto de gatekeeper, para referirnos a categorías, funciones y factores variados; se trata de una consecuencia de la adopción de las conceptualizaciones de la sociología, y en particular de la sociología de la literatura que tiende a topologizar, a espacializar las relaciones en el ámbito cultural en función de posiciones sociales; cuestionarlos implica proponer nuevos modos de relaciones que pongan el acento en los desplazamientos y en la temporalidad.

\section{El caso Caillois}

El caso de Roger Caillois resulta particularmente productivo en parte porque ha sido objeto de numerosos estudios, y produce la impresión de ser conocido. Se lo suele considerar como uno de los más importantes gatekeepers de la literatura hispanoamericana al menos en el período 1951-1970, fundador de la primera colección francesa dedicada exclusivamente a la literatura hispanoamericana, "La Croix du Sud", en Gallimard, cuyo primer volumen se publica en 1951, mientras la colección "Obras representativas" de la UNESCO aparece desde 1948, y el primer volumen de literatura hispanoamericana en 1952. Como es sabido, la colección de Caillois constituyó una puerta de entrada para los autores hispanoamericanos contemporáneos, y él mismo jugó un papel determinante en la selección de autores traducidos y editados. Si tratamos de reconstruir el recorrido que lleva a la peculiar posición de Caillois, observamos no solamente factores diferentes, cuyo significado varía según el período, sino también que son de naturaleza distinta, lo cual dificulta una clasificación.

Recordemos rápidamente el recorrido de Caillois. Su contacto con América Latina se produce a través de su relación, amorosa y amistosa, con Victoria Ocampo, quien lo invita a Argentina a dictar una serie de conferencias en 1939; la guerra estalla, y sus posiciones políticas hacen imposible un retorno a la Francia ocupada, y permanece hasta comienzos de 1945 (Felgine 1991, 1994). Junto con actividades destinadas a ganarse la vida, Caillois desarrolla otras que marcan una continuidad con sus empresas europeas, como la fundación del Institut Français d'Études Supérieurs de Buenos Aires en 1942, con Roberto Weibel-Richard; y otras que, de hecho, le abrirán nuevas perspectivas intelectuales, como la fundación y dirección de la revista Lettres françaises, editada en Buenos Aires entre julio de 1941 y abril de $1945^{1}$, y la publicación de la colección $L a$

1 Caillois deja Buenos Aires el 25 de enero de 1945, y regresa a Francia con la misión Pasteur Valéry-Radot, pasando por Santiago de Chile, Lima, Quito, Nueva York, y Londres. 
Porte Etroite. Si bien Caillois había participado en diferentes publicaciones en Francia, no había dirigido aún empresas editoriales de modo autónomo. La experiencia argentina, por tanto, implicará su iniciación no solamente a la cultura local (con sus límites), sino también a funciones dentro del campo que no había ejercido en su propio país; sus emprendimientos están marcados por el hecho de ser un extranjero en un medio cultural que no es aquel en el que ha sido formado, cuya lengua no domina, y cuya cultura desconoce (Louis 2020c). En 1946, ya de vuelta en Francia, Caillois integra la misión del Ministère des Affaires Etrangères, que lo lleva a las Antillas, México, Guatemala, Colombia, Estados Unidos, durante la cual descubre otras facetas de Latinoamérica: zonas de la cultura que no están determinadas por las redes de Victoria Ocampo, y un tipo de intercambio entre Francia y Latinoamérica que se desarrolla en un nivel oficial y político (Guerrero 2018). Caillois se encuentra luego de ese viaje en posición de elegir entre un puesto de investigador, y uno de funcionario de la UNESCO, y opta por la segunda posibilidad en 1948. En 1951 inaugura su colección en Gallimard, con Ficciones de Jorge Luis Borges, seguido por Doña Bárbara de Rómulo Gallegos (1952). Este rápido recorrido sin embargo no tiene en cuenta una serie de factores que permiten comprender la complejidad de su papel; comienzo por enumerarlos, antes de reflexionar acerca del modo en que interactúan, y sobre cómo se los puede considerar en relación con concepto de gatekeeper.

\section{Factor 1: Redes editoriales francesas}

Las relaciones entre Caillois y Gallimard comienzan en 1937, con el interés de Gaston por la ensayística de Caillois; en 1942, habiendo leído L'Homme et le sacré, el editor le escribe a Buenos Aires para manifestar su interés, y lamentar que no le haya confiado la edición, y lo incita a enviarle otro manuscrito; renueva su interés en una carta que Caillois no recibe, del 18 de julio de 1945, donde lo invita a ir a verlo cuando vuelva a Francia, para discutir sobre la publicación de sus libros, le pide una colección completa de la revista Lettres Françaises y manifiesta el deseo de seguir siendo su editor. El reencuentro que se produce en ese momento lleva a la firma de un contrato el 29 de octubre de 1945 para publicar Impostures de la poésie, Rocher de Sisyphe, Circonstancielles; simultáneamente es nombrado director de La Croix du Sud (Carta de Gaston Gallimard a Roger Caillois del 29 de octubre de 1945, archivos Gallimard). Este Factor, por tanto, va a permitir una asociación privilegiada con un editor prestigioso, pero no es suficiente para otorgarle a Caillois el papel determinante que 
tendrá, porque la idea de una colección dedicada a la literatura hispanoamericana es suya (Correspondance Ocampo-Caillois 1997: 226). Notemos que la entrada y la participación en estas redes están fuertemente condicionadas por factores personales: gustos literarios, simpatías y amistades, sin olvidar que Caillois como opositor del régimen podía ser una ventaja para un editor como Gallimard en la inmediata posguerra.

\section{Factor 2: Redes editoriales argentinas}

Como dijimos, en el caso de Caillois, en un primer momento se trata esencialmente de las redes de Victoria Ocampo, a quien había conocido en París en 1938; la relación amorosa, y luego la amistad, fueron, por tanto, determinantes en la posibilidad de integrar estas redes, puesto que Victoria tenía un control importante sobre las posibilidades financieras necesarias para invitar y publicar autores extranjeros (y argentinos por supuesto); como sabemos, financiará también la revista Lettres françaises. Dentro de esta función de director de la revista, Caillois va a funcionar como un gatekeeper de una red de franceses exilados y/o opuestos al régimen de Vichy a quienes invita o permite publicar en su revista - y a los que paga por ello-, como SUR, lo cual constituyó una ayuda considerable para ciertos escritores que se encontraban en América del Norte o del Sur, y en Francia. En este caso, como vemos, la red se desdobla gracias a la creación de una revista en francés, en las condiciones estudiadas (Louis 2013, 2020b), y sus dos directores, Victoria Ocampo y Caillois, funcionan como gatekeepers, lo cual significa que son quienes seleccionan y convocan, mientras defienden una ideología estética y política; la ideología política es un primer "filtro", porque, aunque las relaciones personales y los acuerdos estéticos siguen siendo importantes, pasan a un segundo plano. Estas dos redes se superponen, y se determinan una a la otra; recordemos que una red es una serie de posibilidades, pero también de límites - de selecciones- de circulaciones y rechazos. Por otra parte, Caillois acepta o decide abrir un espacio a la literatura escrita por hispanoamericanos en francés o traducida al francés (Louis 2020b), haciendo así circular sus textos en la comunidad de exilados franceses.

\section{Factor 3: El conocimiento de la lengua castellana}

Varios testimonios permiten afirmar que Caillois no sabía español al llegar a Argentina, y tenía un conocimiento más que limitado al partir en 1945 (Felgine 
1994: 211). Como he tenido ocasión de sugerirlo, en el rechazo del español de Caillois hay un gesto de posicionamiento de su identidad de exilado (Louis 2013); pero en Argentina se abre para él la posibilidad de un aprendizaje paulatino, que siempre estará vinculado a los libros. Su matrimonio con Yvette Billod en 1940, quien aprende rápidamente español, lo asiste con la publicación de la revista y el resto de sus tareas, pasa la agregación al volver a Francia, permite también explicar por qué no le resulta urgente aprender español; lo que sabemos del matrimonio es suficiente para sugerir que las primeras traducciones de Caillois pueden ser el resultado de un trabajo de colaboración. Pero también podemos suponer que, una vez de vuelta en Francia, sus compatriotas asumen que Caillois aprendió español durante sus cinco años de estadía en Argentina, lo cual lo posiciona ante sus ojos en un lugar privilegiado para introducir una literatura que es aún poco conocida en Francia (Molloy 1972). Esta situación, sin embargo, lo fragilizaba ante los escritores hispanoamericanos, a pesar de que su poder de decisión editorial se mantenía. Recordemos también que la falta de interés por la lengua y la cultura latinoamericana de Caillois en su período argentino, hasta que comprende el capital que su estadía y las suposiciones de sus compatriotas implican, contrasta con su interés por la naturaleza americana; la oposición naturaleza/cultura será, como es sabido, objeto de una violenta polémica con Lévi-Strauss en los años $1950^{2}$.

\section{Factor 4: La lengua}

La lengua en la concepción de Caillois se presenta como un medio, o un prisma: un modo de expresión de algo otro. La identidad cultural por ejemplo; responde, por lo tanto, a la idea de transparencia del lenguaje. Caillois sostendrá que existen estructuras comunes a la naturaleza y a las producciones de los hombres, artísticas en particular; la lengua permite así acceder a imágenes que no sean fortuitas. Esta concepción es esencial en las traducciones efectuadas por Caillois, así como en las polémicas que mantuvo contra el surrealismo (Louis 2020a). Si podemos describir de este modo esquemático su concepción de la lengua francesa, queda la cuestión de su concepción del español en tanto lengua literaria; en "Poètes d'Amérique" (7-8), su introducción al conjunto que reúne en el número 12 de Lettres françaises, compuesto de "Saudade" de Ga-

2 No puedo detenerme aquí en esta polémica que se desarrolla en las etapas siguientes Caillois "Illusions à rebours" (1954); Caillois "Illusions à rebours (fin)” (1955); Lévi-Strauss "Diogène couché” (1955). 
briela Mistral (9-16), y de “Arbres. Arbres dans la nuit et le jour, Pins” de Jules Supervielle (17-20), sostiene que la identidad latinoamericana de estos poetas se encuentra en la relación que mantienen con la naturaleza, como si la lengua en que la se escribe no fuera determinante, un movimiento por el cual la naturaleza se vuelve cultura (Louis 2020b, 2020c). A esto viene a sumarse el hecho que, en sus primeras traducciones del español, las ya mencionadas de Gabriela Mistral y las que incluye en su volumen de 1946, así como en sus primeros textos sobre esta práctica, no encontramos una concepción de la traducción, sino un tipo de ejercicio que recuerda la práctica escolar y académica francesa de la versión, como ya lo señalara Néstor Ibarra (1969: 151-152). Agreguemos que su aprendizaje del español se hizo más bien después de su estadía: traducción y aprendizaje de la lengua se producen simultáneamente, lo que podemos constatar fácilmente en sus primeras traducciones (Louis 2020b).

\section{Factor 5: La concepción de la literatura y la poesía}

La revista Lettres françaises permite a Caillois publicar una serie de ensayos en los cuales se desarrolla y expone su concepción de la literatura, como en "Manifeste pour une Littérature édifiante", en el número $13^{3}$, donde defiende el concepto de "literatura edificante", que se opondría a "literatura de diversión", categorías que sitúa en el nivel del estilo. Ya en su producción anterior había mostrado su desconfianza hacia la poesía y la ficción, y se había pronunciado abiertamente en favor del ensayo (como Victoria Ocampo por otra parte), una tendencia que se pronuncia ante los acontecimientos políticos del período; aunque, como sabemos, es el período en que Sur abre un espacio a la ficción borgeana y contribuye a su desarrollo gracias a la influencia de José Bianco (Louis 2006). En cuanto a su concepción de la lengua poética, como dije, es tributaria de la idea de transparencia del lenguaje, por lo cual los surrealistas aparecen como los artífices de la desnaturalización de lo que considera la función elevada de la poesía, servir a la causa del hombre (Gramuglio 1992). Acerca de la ficción, conviene aclarar que "edificante” significa también que tiene un

3 "Je l'avouerai sans ambages: en général, je n'ai de goût que pour la littérature édifiante. C'est la seule qui me semble atteindre la grandeur. Le reste demeure divertissement; on en fait que s'en distraire. Mais il s'agit de littérature et l'édification propre aux Lettres réside dans le style" (Lo confieso abiertamente: en general, no me gusta más que la literatura edificante. Es la única que me parece alcanzar la grandeza. El resto es entretenimiento, no hacemos sino distraernos. Pero se trata de literatura y la edificación propia a las Letras reside en el estilo) (1944: 2). 
significado explícito, o explicitado en el final del texto al lector - y como lo prueban las traducciones que Caillois hizo de Borges: por el autor o el traductor ${ }^{4}$. Estas concepciones son determinantes en sus elecciones editoriales, pero no son únicas, sino que se superponen a los intereses editoriales y financieros, los gustos personales, y las relaciones y redes.

\section{Factor 6: Las batallas genéricas}

Dos géneros interesan de un modo específico a Caillois en el momento en que se lanza a sus proyectos editoriales en Francia: el policial y lo fantástico. En ambos casos, sus concepciones lo van a oponer no solamente a Jorge Luis Borges sino también a tradiciones en constitución en Latinoamérica, que se desarrollan en esos años, y ganan terreno en el período en que edita "La Croix du Sud" (Louis 2002; Correspondance. Roger Caillois-Victoria Ocampo 1997: 114-115; Louis 2020a: 125-146). Si su interés por el policial se diluye, su trabajo de editor y de traductor, especialmente de Borges, acentúa su inclinación por la literatura fantástica, o viceversa: elige esos autores y textos para traducir porque sigue interesado en lo fantástico. Caillois suele considerar el género a partir de su vertiente europea, y su concepción no corresponde a la naciente práctica del género en América Latina, por lo cual sus elecciones y traducciones atenúan efectos, e introducen cierto grado de explicación en un universo fantástico que suele rechazar toda explicitación. En sus traducciones y ediciones va a inscribir su propia concepción, de modo en parte deliberado, modificando el significado de los textos, o intentando modificarlo. No se trata, como se ha pretendido a menudo, de una falta de comprensión de parte de Caillois de la literatura latinoamericana, sino de una concepción otra, que entra en competencia con la de Borges, Bioy, Cortázar, y con algunos principios del género hispanoamericano. Desde su lugar de traductor y editor, inscribe su propia concepción, usando la traducción, así como la de la antología y del ensayo, para enfrentar las teorías a las que no se adhiere, como podemos verlo en su Anthologie de la littérature fantastique (1965). Caillois no ganó esta batalla (por suerte), la literatura fantástica latinoamericana se impuso con

\footnotetext{
4 En una carta de Julio Cortázar a Jean Barnabé del 8 de mayo de 1957, éste cuenta un encuentro con Caillois en que el francés le propone agregar una frase final al cuento "La noche boca arriba” donde se aclararía al lector francés que no se trata de una alucinación del hombre a quien han operado, y cerrar el cuento diciendo que a la mañana siguiente los enfermeros encontraron muerto al enfermo, y al mirarlo con atención se dieron cuenta de que tenía una herida en el pecho y que le faltaba el corazón (Cortázar 2012: 126).
} 
sus especificidades, pero en los años 1950, 1960, el enfrentamiento de estas concepciones no permite aún saber cuál de estas estéticas se impondrá, y en la batalla librada por Caillois la traducción juega un papel preponderante, así como sus ensayos, en particular en el caso de Borges (Louis 2020a) ${ }^{5}$.

\section{Factor 7: Agente institucional de cultura}

La carrera oficial de Caillois como agente cultural francés comienza, como dijimos, en 1946, cuando realiza la misión para el Ministère des Affaires Etrangères, que le abre las puertas del mundo diplomático francés en América Latina y le permite extender las fronteras de sus redes, y conocer otras facetas de la cultura y de la geografía: un mundo menos francófilo y francófono. En esta misión conoce, por ejemplo, a Miguel Ángel Asturias (Louis 2013). Es sin duda entonces cuando la dimensión política de la difusión cultural se vuelve evidente para él, un aspecto que va a jugar un papel esencial en sus empresas editoriales y será determinante en su decisión de integrar la UNESCO. Caillois participa así en un movimiento de expansión de instituciones en América Latina y de representación del subcontinente en Francia, incluso en las universidades (Guerrero 2018). Esta función, que contrasta con la situación en la que se encontraba durante su estadía en Argentina, de refugiado y opositor del gobierno de Vichy, implica obligaciones, favores y libertades diferentes que la de editor de Gallimard, pero se asemeja a su cargo en la UNESCO, por supuesto, sin confundirse con este. La gestión cultural y política implica la adopción de una forma de representatividad de la nación, por lo cual, si consideramos las elecciones editoriales, incluso las más concretas (un autor, una obra, un traductor ... ), se trata de poner en comunicación dos naciones, dos culturas, en el marco de coyunturas políticas precisas. Sin embargo, la noción de representatividad, como lo ha mostrado Susane Klengel precisamente a propósito de la colección de la UNESCO, es altamente compleja y remite a la de "clásico" (2018); en el caso de Caillois, si se atribuye una obra a la colección “Obras representativas”, podemos preguntarnos qué representan entonces las de la colección "La Croix du Sud" (Bastide 1958, Louis 2013).

5 Es evidente por otra parte que la publicación de la Introduction à la littérature fantastique de Todorov en 1970 va a modificar la percepción del género. Ver Louis (2015: 113-128). 


\section{Factor 8: Recorte editorial francés o panorama editorial francés}

Este factor se diferencia del que concierne a las redes editoriales francesas porque considera la topografía de territorios y la superposición de fronteras editoriales, es decir el modo en que se reparte el campo para ubicar una literatura extranjera. Sin embargo, no se trata, en verdad, de un territorio que se reparte sino que debe ser creado: el espacio editorial de las literaturas extranjeras no existe previamente en un país, debe ser primero imaginado a partir de la literatura nacional, de la recepción de una literatura extranjera por aquellos capaces de leerla en su lengua original, de la concepción de otras literaturas extranjeras. Entre los parámetros que juegan en este caso se encuentran las condiciones concretas de existencia de la colección de Gallimard (presupuesto, medios, grafistas, etc.), la existencia previa de la colección "Du monde entier" en la misma editorial, fundada en 1931, la mencionada colección de la UNESCO. Este territorio se negocia y se reparte a partir de la superposición de varios criterios: la oposición entre literatura francesa y literaturas extranjeras, la relación ambivalente entre obras clásicas/obras representativas de una cultura (Klengel 2018), el intento de definición de un eje específico para una colección de literatura hispanoamericana (Bastide 1958).

\section{Conclusiones y propuestas}

El examen del caso Caillois lleva a postular una diferencia entre agentes y factores. Propongo, por lo tanto, conservar el término de gatekeeper para quienes ejercen una función de preservación de una institución, y de selección, es decir para lo que hoy son los agentes literarios y editoriales, teniendo en cuenta, sin embargo, que se trata de funciones que consisten en una aceptación o negación que ponen en marcha un proceso. Creo que podemos asociar la figura del agente literario-gatekeeper al guardián del célebre relato de Kafka "Ante la ley”, así como a la lectura que Derrida hizo de este, y a la de Josefina Ludmer de ambos: los agentes, independientes o internacionales, custodian una forma de institucionalización de la literatura, sin la cual es imposible el funcionamiento del ambiente (Ludmer 2015: 231-288). Custodiar puede significar tanto ser fiel a ciertos principios como subvertirlos, todo depende de qué es lo que se quiere preservar. Por razones de mercado, los gatekeepers suelen custodiar la institución literaria poniendo el acento en la cultura a la que se traduce, la cultura entrante, y en el efecto que la entrada de un autor y/o de un libro tienen 
en esta; pero puede ocurrir que se apueste por el efecto de extrañeza de una obra y un autor. Sabemos, por otro lado, que las traducciones se proyectan hacia la cultura de origen, porque pueden reposicionar un autor o una obra en su propio campo. Ellas juegan un papel variado en el proceso dinámico de recepción en el marco de la circulación de los autores entre culturas.

Otra conclusión que permite el caso Caillois es que se trata de un gatekeeper de una categoría especial, que podemos llamar “catalizador”. En el campo de la química, un catalizador es un cuerpo o sustancia que provoca una catálisis, que determina y acelera una reacción; sus elementos esenciales son la reacción y la aceleración, y el hecho que el catalizador puede quedar ínfimamente mezclado al producto final. Por lo tanto, el catalizador transforma y es transformado en el proceso. Para volver al caso de Caillois, sus traducciones, ediciones, apropiación mediante antologías de literatura hispanoamericana pueden pensarse en función de los diferentes factores mencionados, pero también en función de la posibilidad de construirse en el campo, y de vehicular su propio proyecto literario: un factor determinante sin dudas, y por momentos dominante, como lo he podido mostrar en el caso de la construcción de la edición y recepción de Borges en Francia mediante la intervención de Caillois (2020a).

Puesto que propongo desglosar la categoría de gatekeeper en diferentes agentes, he aquí algunas de las otras categorías:

- La amistad: algunos amigos pueden participar en la promoción literaria de un autor. Dos casos diferentes pueden presentarse: los amigos que proporcionan un apoyo económico; los amigos que actúan como promotores, es decir que además de sus vínculos personales intentan difundir la literatura de un autor. Puede tratarse de amigos del autor que lo promocionan o de amigos de los editores $u$ otros mediadores;

- El gusto estético y literario, por lo tanto, de los actores que intervienen en el proceso de edición de un autor extranjero, que juega un papel esencial en el caso de autores poco reconocidos mundialmente, porque en estos casos la decisión se toma en función de otros factores que el interés financiero. Numerosas editoriales examinan las propuestas enviadas de forma espontánea por traductores free-lance y/o amateurs, que de este modo influyen en sus políticas editoriales;

- Los lectores, evaluadores y otros mediadores en las etapas de pre-edición: aunque suelen permanecer anónimos, estos lectores y su formación, gustos y concepciones son esenciales, porque tradicionalmente las editoriales confiaban los libros a varios evaluadores (dos por lo general); gustos personales, concepciones literarias y concepción del público al que se dirige la traducción y del mercado resultan determinantes en estas evaluaciones. Todos estos actores tienen un poder de decisión capital, pero sus intereses 
generalmente son de orden editorial y comercial, o, en todo caso, estos son los que predominan (Guerrero 2016).

Estos agentes no son meros mediadores, tanto ellos como los gatekeepers cumplen una función de traslado: llevan un texto de una cultura a otra. Notemos, además, que la dificultad para discriminar estas funciones viene del hecho de que muchos agentes acumulan varias de estas funciones, y cumplen papeles variados. Es el caso de Caillois, reúne al menos las funciones de gatekeeper (selección concreta de autores, inclusión y exclusión), y de catalizador, así como de traductor, lo cual, si consideramos el impacto de las literaturas extranjeras en las nacionales, implica una incidencia fundamental en la historia literaria francesa. Estas diversas funciones asumidas por Caillois permiten un acercamiento distinto a su figura, y también comprender que su trabajo editorial no responde a un único parámetro; lo que aparece como una falta de línea coherente editorial no resulta meramente de la superposición de funciones (editor de Gallimard, funcionario de la UNESCO), sino de la suma de factores que juegan en sus decisiones, y que he intentado describir. La pregunta es si llegar a postular una interpretación coherente es el objetivo de la investigación o si podemos construir sistemas interpretativos que incorporen anomalías, factores múltiples, superposiciones y pluralidades.

\section{Bibliografía}

Bastide Roger (1958): “Sous “La Croix du Sud”: l’Amérique latine dans le miroir de sa littérature”. En: Annales 13/1, pp. 30-46.

Bourdieu, Pierre (1991): “Le champ littéraire”. En : Actes de la Recherche en sciences sociales 89, pp. 4-46.

Bourdieu, Pierre (1966): “Champ intellectuel et projet créateur”. En: Les Temps modernes 246, pp. $865-906$.

Caillois, Roger (1965): Au cœur du fantastique. París: Gallimard.

Caillois, Roger (enero 1955): "Illusions à rebours (fin)". En: NNRF, pp. 58-66.

Caillois, Roger (diciembre 1954): “Illusions à rebours”. En: NNRF, pp. 1010-1024.

Caillois, Roger (01/07/1944): “Manifeste pour une littérature édifiante”. En: Lettres françaises 13, pp.1-5.

Caillois, Roger (01/04/1944): "Poètes d’Amérique”. Lettres françaises 12, pp. 7s.

Cortázar, Julio (2012): Cartas, 5 vol. Madrid: Alfaguara. Eds. Aurora Bernárdez/Carles Álvarez Garriga.

De Certeau, Michel (1990): L'invention du quotidien 1. Arts de faire. París: Gallimard. Felgine, Odile (1994): Roger Caillois (biographie). París: Stock.

Felgine, Odile (1991): Victoria Ocampo. París: Criterion. Préambule d’Ernesto Sábato. 
Felgine, Odile/Ayerza de Castilho, Laura (eds.) (1997): Correspondance. Roger Caillois-Victoria Ocampo. París: Stock.

Foucault, Michel (1975): Surveiller et punir. París: Gallimard.

Foucault, Michel (1971): L'Ordre du discours. París: Gallimard.

Gallego Cuiñas, Ana (julio-agosto 2018): "Las narrativas del siglo XXI en el Cono Sur: estéticas alternativas, mediadores independientes”. En: Insula 859-860, pp. 8-12.

Glass, Loren (2008): “Markets and Gatekeepers”. En: Stoneley, Peter/Weinstein, Cindy (eds): The Blackwell Concise Companion to American Fiction, 1900-1950. Nueva York: Blackwell, pp. 77-93.

Gramuglio, María Teresa (1992): “Roger Caillois en Sur”. En: Río de la Plata 13-14, pp. 149-169.

Guerrero, Gustavo (2018): “La Croix du Sud (1945-1970): Génesis y contextos de la primera colección francesa de literatura latinoamericana”. En: Locane, Jorge/Loy, Benajmin/ Müller, Gesine: Remapping World Literature: Writing, Book Markets and Epistemologies between Latin America and the Global South. Berlín/Boston: De Gruyter, pp. 199-208.

Guerrero, Gustavo (2016). “Mediadores editoriales”. Seminario MEDETLAT, ENS-París, 16/03/ 2016.

Ibarra, Néstor (1969): Borges et Borges. París: L’Herne/Glose.

Klengel, Susanne (2018): “El derecho a la literatura (mundial y traducida). Sobre el sueño traslatológico de la Unesco". En: Locane, Jorge/Loy, Benajmin/Müller, Gesine, Remapping World Literature: Writing, Book Markets and Epistemologies between Latin America and the Global South. Berlín/Boston: De Gruyter, pp. 131-155.

Lévi-Strauss, Claude (marzo 1955): “Diogène couché”. En: Les temps modernes 10/110, pp. 1187-1220.

Louis, Annick (2020a): "El Aleph de Roger Caillois en Gallimard o de cómo salir del laberinto". En: Guerrero, Gustavo/Locane, Jorge/Loy, Benjamin/Müller, Gesine (ed.): Literatura latinoamericana mundial. Dispositivos y disidencias. Berlín/Boston: De Gruyter, pp. 199-208.

Louis, Annick (2020b): “La traduction dans la revue Lettres françaises (1941-1947) de Roger Caillois”. En: Camenen, Gersende/Béhar, Roland (eds.): Scènes de la traduction France/ Argentine. París: Editions Rue d'Ulm (en prensa).

Louis, Annick (2020c): "Premiers pas d'un traducteur-éditeur. Roger Caillois entre Lettres françaises (1941-1945) et La Croix du Sud (1951-1970)”. En: Guerrero, Gustavo/Locane, Jorge J./Loy, Benjamin/Müller, Gesine (eds.): Literatura latinoamericana mundial. Dispositivos y disidencias. Berlín/Boston: De Gruyter, pp. 125-146.

Louis, Annick (2015): “Du Rôle de la délimitation du corpus dans la théorie littéraire. À propos de l'Introduction à la littérature fantastique de Tzvetan Todorov et de la critique littéraire hispano-américaine”. En: Durand, Carine/Raguenet, Sandra (eds.): Entre critique et théorie. L'Amérique Latine: un autre regard sur la littérature. París: Classiques Garnier/ Perspectives comparatistes, pp. 113-128.

Louis, Annick (junio 2013): “Étoiles d'un ciel étranger. Roger Caillois et l'Amérique Latine”. En: Bridet, Guillaume (coord.), Littérature Roger Caillois 170, pp. 71-81.

Louis, Annick (2006): Borges face au fascisme I. Les causes du présent. Montreuil: Aux lieux d'être.

Louis, Annick (2002): “Caillois-Borges, ou qu'est-ce qui s’est passé?”. En: Courtois, JeanPatrice/Krzywkowski, Isabelle (dir.): Diagonales sur Roger Caillois. Syntaxe du monde, paradoxe de la poésie. París: Éditions L’Improviste, pp. 81-101. 
Ludmer, Josefina (2015): Clases 1985. Algunos problemas de teoría literaria.Edición y prólogo Annick Louis. Buenos Aires: Paidós.

Marling, William (2016): Gatekeepers. The Emergence of World Literature and the 1960s. Nueva York: Oxford University Press.

Mistral, Gabriela (1946): Poèmes. París: Gallimard/NRF.

Molloy, Silvia (1972): La diffusion de la littérature hispanoaméricaine en France au XXe siècle. París: PUF.

Sapiro, Gisèle (2007): “L’apport du concept de champ à la sociologie de la littérature”. En: Littérature et sociologie. Bordeaux: Presses Universitaires de Bordeaux, pp. 61-79.

Schlanger, Judith (1992): “Fondation, nouveauté, limites, mémoire”. En: Les débuts des sciences de l'homme, Communications 54, pp. 289-298.

Thompson, John B. (2012): Merchants of Culture: The Publishing Business in the Twenty-First Century. Londres: Penguin.

Topuzian, Marcelo (2014): “La literatura mundial como provocación de los estudios literarios". En: CHUY. Revista de estudios literarios latinoamericanos 1/1, pp. 94-138.

Wijntrep, Lies (2015): Making Borges. The early reception of Jorge Luis Borges's Work in France and the United States. Nijmegen: Radboud University Nijmegen. 
